\title{
Modeling of Precipitation Polymerization I: The Method of Finite Molecular Weight Moments
}

\author{
H. Bouhendi, ${ }^{12}$ V. Haddadi-Asl, ${ }^{13^{*}}$ M. Rafizadeh, ${ }^{1}$ A. Safinejad ${ }^{3}$ \\ ${ }^{1}$ Polymer Engineering Department, Amirkabir University of Technology, P.O. Box \\ $15875-4413$. \\ ${ }^{2}$ Iran Polymer and Petrochemical Institute, P.O. Box 14965/115. \\ $3^{*}$ National Petrochemical Co., Petrochemical Research and Technology Co., P.O. \\ Box 14258, Iran, Tehran. Email: haddadi@aut.ac.ir
}

(Received: 25 July, 2007; published: 4 November, 2007)

\begin{abstract}
Precipitation polymerization method is a non homogeneous method in which polymer chains are separated from continuous phase as polymer particles. In this method, all polymerization reactions occur in continuous and polymer dispersed phases based on simultaneous polymerization theory. Hunkeler theory is a perfect "simultaneous polymerization" theory that predicts molecular weights and the rate of polymerization for precipitation polymerization of hydrophilic monomers. In this paper, finite moment method is used to predict features of precipitation polymerization. This model is able to predict number and weight average degree of polymerization in dispersed and continuous phases, polydispersity index (PDI) for two phases and volume fraction of primary and secondary particles. Experimental results are in agreement with the properties of dispersed phase; weight and number average degree of polymerization in the dispersed phase is more than that of degrees of polymerization in the continuous phase. The PDI of polymer formed in the dispersed phase is equal to 2 and that of PDI in the continuous phase is less than 2. Furthermore Hunkeler mathematical model is reviewed critically.

Keywords: Degree of polymerization, Finite moment, Modeling, Precipitation, Radical polymerization
\end{abstract}

\section{Introduction}

Precipitation polymerization processes in which the produced polymer separates from an initially homogeneous reaction mixture has been used to produce very fine polymeric particles. The rate of precipitation polymerization is faster than the rate of solution polymerization and slower than that of dispersion polymerization. (The size of polymeric particles are controlled by steric stabilizers) [1,2]. Precipitation and dispersion polymerization are categorized as semi homogeneous polymerization. In semi homogeneous polymerizations, reaction is initiated from homogeneous phase (continuous phase) and by adding the initiator, the system becomes heterogeneous as the formed polymer precipitates from solution. After this, the polymerization is assumed mainly to occur in the precipitated polymer phase. According to this assumption, an increase in the volume fraction of the polymer increases the number of loci of polymerization and so the rate of polymerization rises up to a maximum. The increase of the volume fraction of the precipitated polymer also causes an increase in viscosity [1, 2].

In homogeneous polymerization such as solution and bulk polymerization, all polymerization reactions (initiation, propagation and termination) take place in the 
homogeneous phase [3, 4]. In non homogeneous (heterogeneous) polymerization such as suspension and emulsion polymerization the reaction medium is in two phases (dispersed and continuous phase). In suspension polymerization all polymerization reactions take place in the dispersed phase but in emulsion polymerization propagation and termination take place in dispersed phase and initiator decomposition take places in continuous phase $[5,6]$.

In precipitation and dispersion polymerization, there are four theories (model) for loci of the polymerization reactions; homogeneous solution polymerization, surface polymerization, interior polymerization and simultaneous polymerization theories.

In solution polymerization theory, initiation, propagation and termination reactions will occur predominantly in continuous phase and no reaction takes place in the dispersed phase [7, 8].

Soini et al $[9,10]$ have presented a mechanism for dispersion polymerization of styrene and methyl methacrylate. They have neglected reactions within precipitated polymer particle if the insoluble polymer volume fraction is less than 0.1 . Then the medium is good solvent for the monomer and the initiator. The polymerization reactions occur in continuous phase and termination by precipitation dominates bimolecular termination.

In 'surface polymerization` theory, initiation take place in the continuous phase but propagation and termination occur in the thin layer on the surface of the polymer particles [11].

Interior polymerization theory is the most popular theory that predicts the features of precipitation polymerization. This theory is based on the following assumption [12]:

- The dispersed phase is formed immediately after the addition of initiator.

- Initiation of polymerization occurs in the continuous phase.

- Chain propagation and termination take place in the dispersed phase.

- The concentration of monomer in the continuous phase is equal to its concentration in the dispersed phase.

Avela et al $[13,14]$ have presented a mathematical model capable of experimentally determining rate of polymerization based on the model developed by Barret [15].

Desimone et al [16] and Muller et al [17] have calculated number and weight average molecular weight based on the method of conventional molecular weight moment. They have assumed that polymerization reactions occur mainly at dispersed phase.

Simultaneous polymerization model suggests that, polymerization reactions occur simultaneously in both dispersed and continuous phases. This mechanism can be divided into a series of reactions in the both continuous and dispersed phases as well as some mass transfer and partitioning steps. Yasuda et al.[18] used this model for feature prediction of dispersion polymerization of styrene in ethanol. This model has been used for dispersion polymerization of methyl methacrylate in $\mathrm{scCO}_{2}$ [19].

The perfect simultaneous polymerization model that we used in this paper has been presented by Hunckeler et al [20]. This model has been used to describe many features of precipitation polymerization of acrylic acid, including the rate of polymerization and the weight average molecular weight. In Hunkeler honor, we rename this ideal model as Hunkeler model (theory). They have presented a mechanism and kinetic modeling for precipitation polymerization of water soluble 
monomers such as acrylic acid and acrylamide. They have calculated instantaneous number and weight average molecular weight by the following equations:

$\bar{M}_{n}=\frac{M_{0}}{\tau} \quad \bar{M}_{w}=\frac{2 M_{0}}{\tau}$

and $\tau=\frac{k_{t p} R_{p}}{k_{p p}^{2}}+\frac{k_{t r, M}}{k_{p p}}+\frac{k_{t r, S}}{k_{p p}}$

where $k_{t p}$ and $k_{p p}$ are pseudo-rate constants for termination and propagation reactions.

and the rate of polymerization is calculated by the following equation;

$R_{P}=\frac{k_{t p}^{0.5} \gamma}{k_{t t}} \frac{\left(2 k_{d}^{\prime} \alpha[I]\right)^{0.5}[M]^{1.5}\left(1-p^{k c r}\right)^{0.5}}{\delta^{0.5}}$

where $\alpha, \delta, \gamma$ are group parameters including the initiator, macroradical and monomer partition coefficients and the volume fraction of dispersed phase. $p$ and $k c r$ are probability of propagation reaction and critical chain length for precipitation.

Hunkeler has compared modeling and experimental results (weight average molecular weight and monomer conversion) and is able to predict overall weight average molecular weight of system.

In this paper, we used finite molecular weight moment for calculating macroradicals and dead polymer concentration and the number - and weight average degree of polymerization in the continuous phase and conventional moment method for calculating number-and weight average degree of polymerization in the dispersed phase and compared the model with experimental results.

\section{Model}

\section{Hints}

The polymerization reaction occurs isothermally and at constant volume. The chain transfer reactions to polymer and initiator are abandoned in both dispersed and continuous phases [20] in this article.

Reactions in continuous and dispersed phase are;

$$
\begin{array}{ll}
I_{j} \stackrel{k_{d j}}{\longrightarrow} 2 R_{0, j} & \text { Initiator decomposition } \\
I_{j}+M_{j} \stackrel{k_{d j}^{\prime}}{\longrightarrow} R_{1, j}^{*}+R_{0, j}^{*} & \begin{array}{l}
\text { Monomer enhanced decomposition of } \\
\text { initiator }
\end{array} \\
R_{0, j}^{*}+M_{j} \stackrel{k_{p j}}{\longrightarrow} R_{1, j}^{*} & \text { Initiation } \\
R_{i, j}^{*}+M_{j} \stackrel{k_{p j}}{\longrightarrow} R_{i+1, j}^{*} & \text { Propagation } \\
R_{i, j}^{*}+M_{j} \stackrel{k_{t r, M j}}{\longrightarrow} R_{1, j}^{*}+P_{i} & \text { Transfer to monomer } \\
R_{i, j}^{*}+M_{j} \stackrel{k_{t r, S j}}{\longrightarrow} R_{1, j}^{*}+P_{i} & \text { Transfer to solvent }
\end{array}
$$


$R_{i, j}^{*}+R_{r, j}^{*} \stackrel{k_{t d j}}{\longrightarrow} P_{i}+P_{r}$

$R_{i, j}^{*}+R_{r, j}^{*} \stackrel{k_{\mathrm{tcj}}}{\longrightarrow} P_{i+r}$
Termination by disproportionation

Termination by combination

where $I, M, R_{0}^{*}, R_{i}^{*}, S$ and $P_{i}$ stand for initiator, monomer, primary radicals, macroradicals containing $i$ monomer unit, solvent and dead polymer containing $i$ monomer unit respectively. $j$ refers to " $d$ " for dispersed and " $c$ " for continuous phases.

According to Hunkeler theory, it is reasonable to assume that the rate constants for the continuous and dispersed phases are the same, i.e. $k_{p c}=k_{p d}=k_{p}$.

Mass transfer between continuous and dispersed phases is as follows:
$I_{c} \stackrel{\varphi_{I}}{\longleftrightarrow} I_{d}$
Initiator
$M_{c} \stackrel{\varphi_{M}}{\longleftrightarrow} M_{d}$
Monomer
$P_{c} \stackrel{\varphi_{P}}{\longleftrightarrow} P_{d}$
Polymer
$R_{0, c}^{*} \stackrel{\varphi_{R_{0}^{*}}}{\longrightarrow} R_{0, d}^{*}$
Primary radical
$R_{C}^{*} \stackrel{\varphi_{R^{*}}}{\longrightarrow} R_{d}^{*}$
Macroradical

where $\varphi$ is the partition coefficient between continuous and dispersed phases. The equation for precipitation of macroradicals in the continuous phase is given by:

$R_{c, k c r-1}^{*}+M_{c} \stackrel{k_{p}}{\longrightarrow} R C_{c, k c r}^{*} \stackrel{k_{d}}{\longrightarrow} R_{d, k c r}^{*} \stackrel{k_{t}^{*}}{\longrightarrow}$ Inert

where $k c r$ is critical chain length for solubility of macroradicals in the continuous phase. This equation is for termination reaction of macroradicals in the continuous phase.

\section{Mass Balance Equations}

There are two differential equations describing the conservation of the monomer and initiator in the system (dispersed and continuous phases).

In the polymerization of water soluble monomers such as acrylamide and acrylic acid, the rate constant for the monomer enhanced decomposition of initiator, $k_{d}^{\prime}$ is much greater than the thermal decomposition constant of initiator, $k_{d}$. The rate of monomer-enhanced decomposition of initiator is at least 15 times larger than the rate of thermal decomposition [20]. Then the rate of initiator consumption can be written as;

$$
\frac{d[I]}{d t}=-\left(k_{d}^{\prime}[I]_{c}[M]_{c} \phi_{c}+k_{d}^{\prime}[I]_{d}[M]_{d} \phi_{d}\right)
$$

where $\phi_{c}$ and $\phi_{d}$ are volume fractions of the dispersed and continuous phases.

Eiler's method was used for calculating volume fraction of the dispersed phase. This method postulates two kinds of particles with different rheological behaviors. Primary 
particles, which are formed directly by precipitation, are the first kind. The second kind are secondary particles which are formed from the primary particles by agglomeration according to a first- order reaction rate equation. The rates of formation for the first and second kind of particles are;

$$
\begin{aligned}
& \frac{d \phi_{1}}{d t}=\frac{d p}{d t}[M]_{0} V_{P}^{\prime}-k_{v} \phi_{1} \\
& \frac{d \phi_{2}}{d t}=k_{v} \phi_{1}
\end{aligned}
$$

In Equations (4) and (5), $V_{P}^{\prime}, k_{v}$, and $p$ are molar volume of polymer, rate constant of change of the kind of particle and conversion respectively. The volume fraction of the dispersed phase (precipitated polymer) is the sum of the volume fractions of the first and the second kind of particles.

$$
\phi_{d}=1-\phi_{c}=\phi_{1}+\phi_{2}
$$

The monomer is consumed by propagation and chain transfer to monomer reactions in both continuous phase and by polymer particles. The consumption rate of monomer is given by:

$$
\frac{d[M]}{d t}=-\left\{k_{p}[M]_{c}[R]_{c} \phi_{c}+k_{p}[M]_{d}[R]_{d} \phi_{d}\right\}-\left\{k_{t r, M}[M]_{c}[R]_{c} \phi_{c}+k_{t r, M}[M]_{d}[R]_{d} \phi_{d}\right\}
$$

In Equation (3) and (7), ' $c$ ' and ' $d$ ' denote the concentration of reactants in the continuous and dispersed phases and are calculated by Equations (8) and (9) as follows:

$$
\begin{aligned}
& {[\beta]_{c}=\frac{\varphi_{i}[\beta]}{\varphi_{i} \phi_{c}+\phi_{d}}} \\
& {[\beta]_{d}=\frac{[\beta]_{c}}{\varphi_{i}}}
\end{aligned}
$$

where $\beta$ corresponds $R_{\text {in }}^{*}, R^{*}, M, P$, and $I$.

\section{Moment equations}

\section{-Equations for continuous phase}

For deriving finite moment equations, the following rate expressions for macroradicals and dead polymer chains were derived.

$$
\frac{d\left[R_{1}\right]}{d t}=\Re_{i}-\left(k_{p}[M]+k_{t r, M}[M]\right)\left(R_{1}\right]-k_{t}\left[R_{1}\right][R]
$$

where $[R]=\sum_{i=1}^{m}\left[R_{i}\right], \quad k_{t}=k_{t c}+k_{t d}$ and $\Re_{i}=2 k_{d}^{\prime}[I][M]$ is the rate of the initiation reaction.

$$
\frac{d\left[R_{i}\right]}{d t}=k_{p}[M]\left(\left[R_{i-1}\right]-\left[R_{i}\right]\right)-k_{t r, M}[M]\left[R_{i}\right]-k_{t}\left[R_{i}\right][R] \quad i \geq 2
$$


$\frac{d\left[P_{i}\right]}{d t}=k_{t r, M}[M]\left[R_{i}\right]+k_{t d}\left[R_{i}\right][R]+\frac{1}{2} k_{t c} \sum_{j=1}^{i}\left[R_{j}\right]\left[R_{i-j}\right] \quad i \geq 2$

When the quasi steady state approximation is applied for primary and macroradicals, one may have:

$\left[R_{1}\right]=(1-\varsigma)[R]$

$\left[R_{i}\right]=\varsigma\left[R_{i-1}\right]$

where $\varsigma$ is the probability of propagation defined as [21]:

$$
\varsigma=\frac{R_{P}}{R_{P}+\sum R_{t r}}
$$

or

$\varsigma=\left\{1+\frac{k_{t r, M}}{k_{p}}+\frac{k_{t r, S}[S]}{k_{p}[M]}+\frac{k_{t}[R]}{k_{p}[M]}\right\}^{-1}$

Rewriting Equation (14) for $R_{i-1}, R_{i-2}, \ldots$, one may obtain:

$\left[R_{i}\right]=\varsigma^{i-1}\left[R_{1}\right]$

and

$\left[R_{i}\right]=\varsigma^{i-1}(1-\varsigma)[R]$

From Equations (13)-(17), Equations (10)-(12) can be rewritten as:

$$
\begin{aligned}
& \frac{d\left[R_{1}\right]}{d t}=\mathfrak{R}_{i}-\left(k_{p}+k_{t r, M}\right)[M](1-\varsigma)[R]-k_{t}(1-\varsigma)[R]^{2} \\
& \frac{d\left[R_{i}\right]}{d t}=\left[k_{p}[M]\left(\varsigma^{-1}-1\right)-k_{t r, M}[M]-k_{t}[R]\right](1-\varsigma) \varsigma^{i-1}[R] \\
& \frac{d\left[P_{i}\right]}{d t}=\left[k_{t r, M}[M]+\frac{1}{2} k_{t c}\left(\varsigma^{-1}-1\right)(i-1)+k_{t d}[R]\right](1-\varsigma) \varsigma^{i-1}[R]
\end{aligned}
$$

For calculating number - and weight average degree of polymerization of polymer formed in the continuous phase, the finite molecular weight moments are defined as:

$$
\begin{array}{ll}
\frac{d \lambda_{j}^{*}}{d t}=\sum_{i=1}^{m} \frac{d i^{j}\left[R_{i}\right]}{d t}=\sum_{i=n}^{m} \frac{d i^{j}\left[R_{i}\right]}{d t}+\frac{d\left[R_{1}\right]}{d t} & \text { for macroradicals } \\
\frac{d \mu_{j}^{*}}{d t}=\sum_{i=n}^{m} \frac{d i^{j}\left[P_{i}\right]}{d t} & \text { for dead polymers }
\end{array}
$$

where $n=2, m=k c r$ and $\dddot{ }$ ' is the moment order. Then the dynamic moment equations can be derived as follow:

$$
\begin{aligned}
& \left.\frac{d \lambda_{0}^{*}}{d t}=\Re_{i}+\left[-k_{p}[M]\left(\varsigma^{-1}-1\right)+k_{t r, M}[M]+k_{t}[R]\right] R\right]\left(\varsigma^{m-1}-\varsigma^{n-1}\right) \\
& -\left[k_{p}[M]+k_{t r, M}[M]+k_{t}[R]\right](1-\varsigma)[R]
\end{aligned}
$$




$$
\begin{aligned}
& \frac{d \lambda_{1}^{*}}{d t}=\Re_{i}+\left[k_{p}[M]\left(\varsigma^{-1}-1\right)+k_{t r, M}[M]+k_{t}[R]\right]\left\{\varsigma^{n}\left[n+(1-\varsigma)^{-1}\right]-\varsigma^{m}\left[m+(1-\varsigma)^{-1}\right]\right\} \\
& -\left[k_{p}[M]+k_{t r, M}[M]+k_{t}[R]\right](1-\varsigma)[R] \\
& \frac{d \lambda_{2}^{*}}{d t}=\left[-k_{p}[M]\left(\varsigma^{-1}-1\right)+k_{t r, M}[M]+k_{t}[R]\right] \\
& \times\left\{\varsigma^{m}\left[m^{2}+2(1-\varsigma)^{-1}\left(m+(1-\varsigma)^{-1}\right)\right]-\varsigma^{n}\left[n^{2}+2(1-\varsigma)^{-1}\left(n+(1-\varsigma)^{-1}\right)\right]\right\} \\
& +\Re_{i}-\left(k_{p}+k_{t r, M}\right)[M](1-\varsigma)(R]-k_{t}(1-\varsigma)[R]^{2}
\end{aligned}
$$

and

$$
\begin{aligned}
& \left.\frac{d \mu_{0}^{*}}{d t}=\left[k_{t r, M}[M]+k_{t d}[R]\right] R\right]\left(\varsigma^{n-1}-\varsigma^{m-1}\right) \\
& +\frac{1}{2} k_{t c}[R]^{2}\left(\varsigma^{-1}-1\right)\left\{\varsigma^{n-1}\left[n-1+(1-\varsigma)^{-1}\right]-\varsigma^{m-1}\left[m-1+(1-\varsigma)^{-1}\right]\right\} \\
& \left.\frac{d \mu_{1}^{*}}{d t}=\left[k_{t d}[R]+k_{t r, M}[M]\right]\left\{\varsigma^{n}\left[n+(1-\varsigma)^{-1}\right]-\varsigma^{m}\left[m+(1-\varsigma)^{-1}\right]\right\} R\right] \varsigma^{-1} \\
& +\frac{1}{2} k_{t c}[R]^{2}(1-\varsigma) \varsigma^{-2}\left\{\begin{array}{l}
\varsigma^{n}\left[n^{2}+\left(2(1-\varsigma)^{-1}+1\right)\left(n+(1-\varsigma)^{-1}\right)\right] \\
-\varsigma^{m}\left[m^{2}+\left(2(1-\varsigma)^{-1}+1\right)\left(m+(1-\varsigma)^{-1}\right)\right]
\end{array}\right\} \\
& \left.\frac{d \mu_{2}^{*}}{d t}=\left(k_{t r, M}[M]+k_{t d}[R]\right)\left\{\begin{array}{l}
\varsigma^{n}\left[n^{2}+2(1-\varsigma)^{-1}\left(n+(1-\varsigma)^{-1}\right)\right] \\
-\varsigma^{m}\left[m^{2}+2(1-\varsigma)^{-1}\left(m+(1-\varsigma)^{-1}\right)\right.
\end{array}\right]\right\} \\
& +\frac{1}{2} k_{t c}[R]^{2}(1-\varsigma) \varsigma^{-1}\left\{\begin{array}{l}
\varsigma^{n}\left\{n^{3}+\left(3(1-\varsigma)^{-1}+1\right)\left[n^{2}+2(1-\varsigma)^{-1}\left(n+(1-\varsigma)^{-1}\right)\right]\right\}- \\
\left.\varsigma^{m}\left\{m^{3}+\left(3(1-\varsigma)^{-1}+1\right)\left[m^{3}+2(1-\varsigma)^{-1}\left(m+(1-\varsigma)^{-1}\right)\right]\right\}\right\}
\end{array}\right.
\end{aligned}
$$

In Equations (15-b) and (23)-(28), $[M]$ and $[R]$ are monomer and macroradicals concentrations in the continuous phase.

Equations in the dispersed phase

Case I:

According to Hunkeler theory [20], termination by disproportionation (or combination) dominates over termination by precipitation mechanism in the dispersed phase. Therefore there is no limitation for radical propagation at this phase. For calculating moment equations for dispersed phase, the conventional method was used. In this method, the total number chain length distribution of macroradicals and dead polymer chains are defined as

$$
\begin{aligned}
& \frac{d \lambda_{0, d}}{d t}=2 k_{d}^{\prime}[M]_{d}[I]_{d}-k_{t d} \lambda_{0, d}^{2} \\
& \frac{d \lambda_{1, d}}{d t}=2 k_{d}^{\prime}[M]_{d}[I]_{d}+k_{p}[M]_{d} \lambda_{0, d}-k_{t d} \lambda_{1, d} \lambda_{0, d}-k_{t r, M}[M]_{d}\left(\lambda_{1, d}-\lambda_{0, d}\right) \\
& \frac{d \lambda_{2, d}}{d t}=2 k_{d}^{\prime}[M]_{d}[I]_{d}+k_{p}[M]_{d}\left(2 \lambda_{1, d}+\lambda_{0, d}\right)-k_{t d} \lambda_{2, d} \lambda_{0, d}-k_{t r, M}[M]_{d}\left(\lambda_{2, d}-\lambda_{0, d}\right)
\end{aligned}
$$




$$
\begin{aligned}
& \frac{d \mu_{0, d}}{d t}=k_{t d} \lambda_{0, d}^{2}+k_{t r, M}[M]_{d} \lambda_{0, d} \\
& \frac{d \mu_{1, d}}{d t}=k_{t d} \lambda_{1, d} \lambda_{0, d}+k_{t r, M}[M]_{d} \lambda_{1, d} \\
& \frac{d \mu_{2, d}}{d t}=k_{t d} \lambda_{2, d} \lambda_{0, d}+k_{t r, M}[M]_{d} \lambda_{2, d}
\end{aligned}
$$

\section{Case II:}

According to Yasuda study [18] for precipitation polymerization of styrene in ethanol, termination by precipitation mechanism dominates bimolecular termination in both phases. Therefore, Equations (23) to (28) were used for moment calculation in the dispersed phase.

By solving Equations (3), (4), (5), (7) and (23)-(28), the number - and weight average degree of polymerization of polymer formed in both continuous and dispersed phase were then calculated by:

$$
\begin{aligned}
& \bar{X}_{n, j}=\frac{\mu_{1}^{*}}{\mu_{0}^{*}} \\
& \bar{X}_{w, j}=\frac{\mu_{2}^{*}}{\mu_{1}^{*}}
\end{aligned}
$$

And at the end, the monomer conversion was calculated by:

$$
p=\frac{[M]-[M]_{0}}{[M]_{0}}
$$

MATLAB software (Version 7.04) was used to solve differential equations of article. Figures of this paper were plotted by MATLAB software too.

Kinetic parameters

Tab. 1. Kinetic constants.

\begin{tabular}{cccccc}
\hline Parameter & Value & Ref. & Parameter & Value & Ref. \\
\hline$k_{d}^{\prime}$ & $13.2 \times 10^{-3} \mathrm{I} / \mathrm{mol}$. Min & 22 & {$[M]_{0}$} & $\begin{array}{c}1.252 \\
\mathrm{~mol} / \mathrm{lit}\end{array}$ & - \\
$k_{p}$ & $108 \times 10^{3} \mathrm{I} / \mathrm{mol}$. Min & $\begin{array}{c}13,23, \\
24\end{array}$ & {$[I]_{0}$} & $8.5 \mathrm{mmol} / \mathrm{lit}$ & - \\
$k_{t d}$ & $58.8 \times 10^{4} \mathrm{I} / \mathrm{mol}$. Min & 20,22 & $V_{P}$ & $0.052 \mathrm{l} / \mathrm{mol}$ & 13,14 \\
$k_{t r, M}$ & $162 \mathrm{l} / \mathrm{mol}$. Min & 20,22 & $\varphi_{I}$ & 1 & - \\
$k_{v}$ & $0.18 \times 10^{-3} \mathrm{~S}^{-1}$ & 13,14 & $\varphi_{M}$ & 1 & - \\
$k_{t c}$ & 0 & 20,22 & $\varphi_{R^{*}}$ & 1 & - \\
$k_{t r, M}$ & 0 & 20,22 & $k c r$ & 2081 & 20,22 \\
\hline
\end{tabular}


The desired kinetic constants and other parameters for precipitation polymerization of acrylic acid in toluene are listed in Table 1 [13, 14, 20, 22, 23, 24].

\section{Results and Discussions}

\section{The justification for the use of finite moment method}

The method of conventional molecular moment is used to calculate number-and weight average degree of polymerization in the homogeneous and heterogeneous methods $\left(\bar{X}_{n}=\frac{\mu_{1}}{\mu_{0}}\right.$ and $\left.\bar{X}_{w}=\frac{\mu_{2}}{\mu_{1}}\right)$ where $\mu_{0}, \mu_{1}$ and $\mu_{2}$ are zero, first and secondary moments of polymer chains. Conventional moments are calculated by the following equation.

$$
\mu_{j}=\sum_{i=1}^{\infty} i^{j}\left[P_{i}\right]
$$

where ' $j$ is the moment order. In the homogeneous polymerization methods, where initiation, propagation and termination reactions take place in a media and in the heterogeneous method where propagation and termination reaction occur in the dispersed phase, the upper boundary for Equation (38) can be infinity, because macroradicals are able to propagate until the termination reaction

In the precipitation and dispersion polymerization, termination by precipitation limits propagation of macroradicals in the continuous phase. In fact the concentration of polymer chains with $i>k c r$ is zero in the continuous phase. Therefore, the conventional moment method (Equation 38) must be converted to the finite moment method as follows;

$\mu_{j}^{*}=\sum_{i=1}^{k c r} i^{j}\left[P_{i}\right]$

Crowley et al $[25,26]$ have used this method for calculating the first and secondary moment of dead polymers in the free radical polymerization of styrene.

Fig. 1 shows monomer conversion versus polymerization time. According to Fig 1 this model (case I and II) predicts approximately the experimental results (Table 2). Same as Hunkeler mathematical model [20], this model has a deviation from experimental results especially at monomer conversions between 0.6 and 0.9 . This deviation is due to purification process of product before gravimetric method. Monomer conversion is calculated by the gravimetric method, in free radical polymerizations, and there are some oligomers that are dissolved in the nonsolvent and go out from the polymerization media during purification process of polymer products. The amount of oligomers (specially the polymer chains lower than $k c r$ ) in the precipitation radical polymerization is more than that of oligomers in homogeneous polymerizations because termination by precipitation dominates bimolecular termination. Therefore, there are some dissolved polymer chains in continuous phase that may go out from product during purification process. Then, gravimetric method is able to calculate the yield of reaction instead of monomer conversion in precipitation polymerization. Therefore, it is possible to calculate yield of reaction by means of gravimetric method. It is assumed that polymer particles (dispersed phase) and polymer chains with critical chain length in the continuous phase cannot be dissolved in the diluent during purification process of product. Hence, there are some oligomers 
in the dispersed phase that cannot be removed from this phase because they are occluded in the polymer particles. Then the yield of reaction is defined as:

yield $=\frac{\left(\phi_{d} \mu_{1, d}+\phi_{c} \sum_{k c r-1}^{k c r} i P_{i}\right)}{[M]_{0}}$

Deviation of monomer conversion from experimental results in case I was more than that of deviation in case II. In case I, there is no limitation for macroradical propagation in the dispersed phase. Then, case I has more dissolved polymer chains with $i>k c r$ than case II. These long chains do not dissolve in the diluent and exits during purification process.

Tab. 2. The result of experiments; $[\mathrm{l}]_{0}=8.5 \mathrm{mmol} / \mathrm{lit}$. and $[\mathrm{M}]_{0}=1.252 \mathrm{~mol} / \mathrm{lit}$.

\begin{tabular}{cccccccc}
\hline $\begin{array}{c}\text { Time } \\
(\mathrm{min})\end{array}$ & yield & $\begin{array}{c}{[\eta]_{1}} \\
(\mathrm{ml} / \mathrm{g})\end{array}$ & $\begin{array}{c}\bar{M}_{n} \\
(\mathrm{~g} / \mathrm{mol})\end{array}$ & $\bar{X}_{n}$ & $\begin{array}{c}{[\eta]_{2}} \\
(\mathrm{ml} / \mathrm{g})\end{array}$ & $\begin{array}{c}\bar{M}_{w} \\
(\mathrm{~g} / \mathrm{mol})\end{array}$ & $\bar{X}_{w}$ \\
\hline 20 & 0.61 & 248.3 & 47088 & 654 & 36.6 & 87120 & 1210 \\
30 & 0.70 & 248.3 & 47088 & 654 & 36.6 & 87120 & 1210 \\
50 & 0.92 & 243.9 & 46152 & 641 & 36.4 & 86400 & 1200 \\
60 & 0.95 & 240.5 & 45432 & 631 & 36.1 & 84816 & 1178 \\
80 & 0.99 & 239.0 & 45144 & 627 & 35.8 & 83520 & 1160 \\
\hline
\end{tabular}

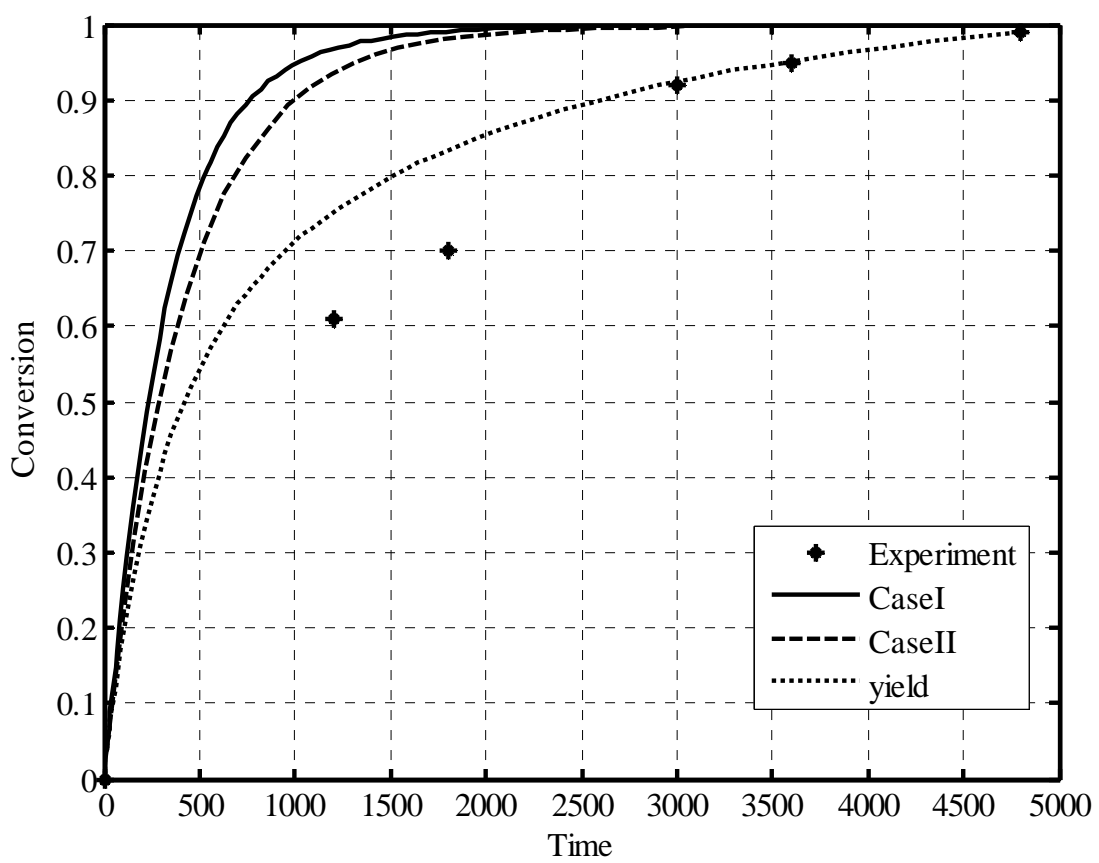

Fig. 1. Monomer conversion versus polymerization time. *Experimental results, Case I, --- Case II , ... yield.

The deviation between this model and experimental results is decreased at the end of polymerization period because the polymerization occurs mainly in dispersed phase at high conversions, and then the concentration of oligomers is decreased by 
increasing monomer conversion in the continuous phase. The polymer chains with $i>k c r$ in the dispersed phase are occluded in polymer particles [1].

Fig. 2 shows weight average degree of polymerization of polymer formed in the dispersed and continuous phases as a function of conversion. The vertical lines at zero conversion are due to zero initial condition for polymer concentration in the differential equations (32) - (34).

Moment methods calculates cumulative weight average and number average degree of polymerization but Hunkeler mathematical model only calculates instantaneous number average degree of polymerization. Experimental properties are cumulative properties and so the comparison between instantaneous properties of Hunkeler model and experimental properties in reference 20 need some improvement.

This model is able to calculate separately degree of polymerization of polymer formed in the both dispersed and continuous phases, but mathematical models have calculated overall number -and weight average degree of polymerization. Other studies [7-19] have also calculated feature of polymerization in either dispersed or continuous phases. According to Fig. 2, experimental results are fitted with weight average degree of polymerization of polymer formed in the dispersed phase (product) in case II but they are not fitted with that of degree of polymerization in the continuous phase. Polymer chains in the continuous phase are removed from reaction media during purification process and are not measured in experimental study.

According to Fig. 2, case I is able to predict experimental results but case II is not. Weight average degree of polymerization of polymer formed in the dispersed and continuous phases are equal in case II.
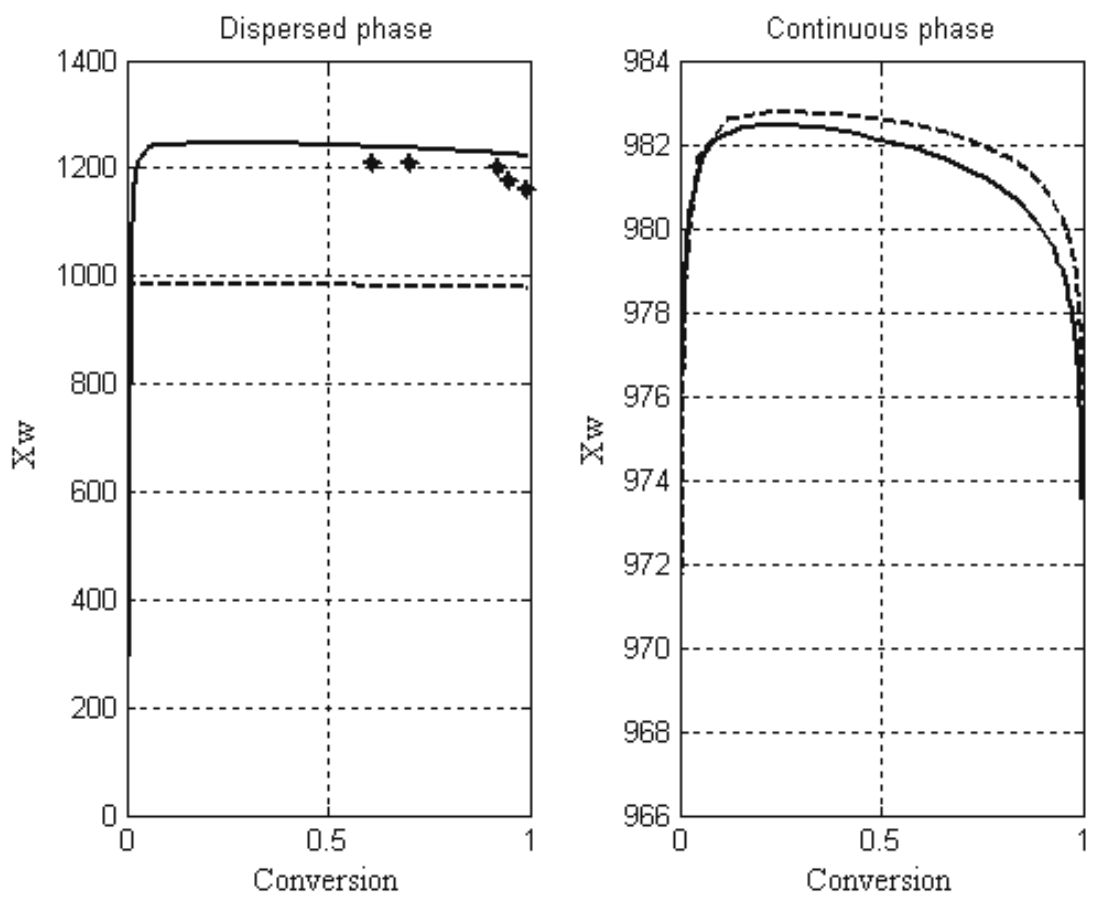

Fig. 2. Weight average degree of polymerization versus monomer conversion. *Experimental results, - Case I, --- Case II 
Fig. 3 shows weight average degree of polymerization for continuous phase. The weight average degree of polymerization of polymer formed in the continuous phase is about 982; this value is less than that of degree of polymerization in the dispersed phase because of termination by precipitation in the continuous phase.

According to Hunkeler theory, number average molecular weight has been calculated by a Mayo like equation (Equations (1-a) and (2)). That model has assumed that PDI $=2\left(k_{t c}=0\right)$ and then weight average molecular weight has been calculated by Equations (1-b). In our study, number and weight average degree of polymerization are calculated separately and then 'PDI' is calculated. Fig. 3 and 4 show number average degree of polymerization and PDI of polymer formed in both phases as a function of conversion.
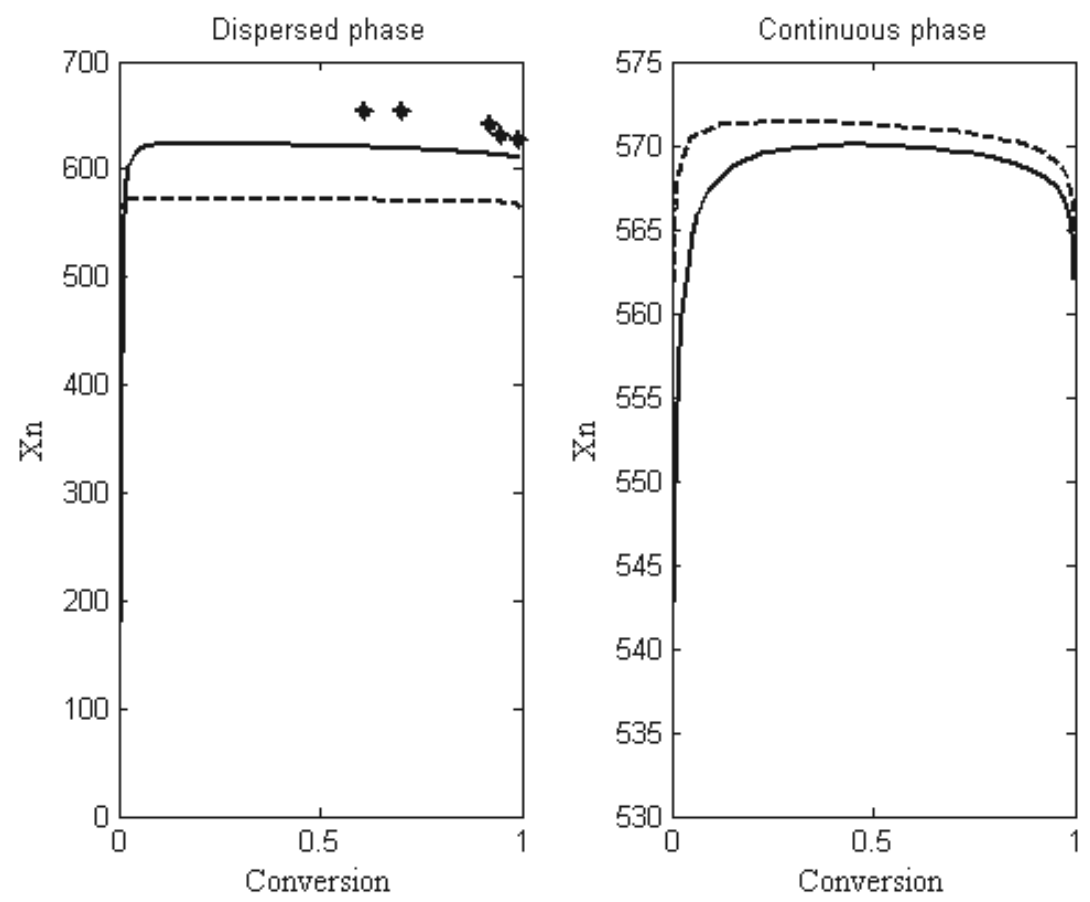

Fig. 3. Number average degree of polymerization versus monomer conversion. *Experimental results, - Case I, --- Case II

According to Fig. 3, number average degree of polymerization of polymer produced in the both continuous and dispersed phase are similar in case II, as, all macroradicals terminate at chain length ' $k c r$ ' at both phases and case II use similar differential equations for calculating degree of polymerization in both phases. Like the weight average degree of polymerization, number average degree of polymerization in case I is fitted with experimental results, this value is 615 for dispersed and 545 for continuous phase. As a result, case II is not reasonable prediction for precipitation polymerization of water soluble monomers such as acrylic acid in toluene.

In homogeneous polymerization weight- and number average degree of polymerization was often decreased rapidly by increasing reaction time. According to mayo equation [21] (Eq. 40), number average degree of polymerization was decreased during polymerization time due to the decreasing instantaneous monomer concentration.

$\frac{1}{\bar{X}_{n}}=\frac{k_{t}}{k_{p}^{2}} \frac{R_{P}}{[M]^{2}}+C_{M}+C_{S} \frac{[S]}{[M]}+C_{I} \frac{[I]}{[M]}$ 
Where $C_{M}, C_{I}$ and $C_{S}$ are relative chain transfer constant for the monomer, initiator and any other compound ' $S$ ' in the reaction system.

In this study, the number- and weight average degree of polymerization is only slightly dependent on the reaction time (Figures 2 and 3 ) because, propagation and termination take place simultaneously in dispersed and continuous phases; in the continuous phase, not only bimolecular termination but also termination by precipitation occurs during polymerization period. Terminations by precipitation cause polymer chains with similar chain length $(k c r)$ to be formed in continuous phase. These polymer chains are transferred to the dispersed phase and form product particles. Termination by precipitation dominates over bimolecular termination in precipitation polymerization of acrylic acid and acrylamide [20], then the number average and weight average degree of polymerization is only slightly dependent on the reaction time. There is no explanation for intense decrease of calculated number and weight average degree of polymerization at high monomer conversion $(P \approx 1)$.
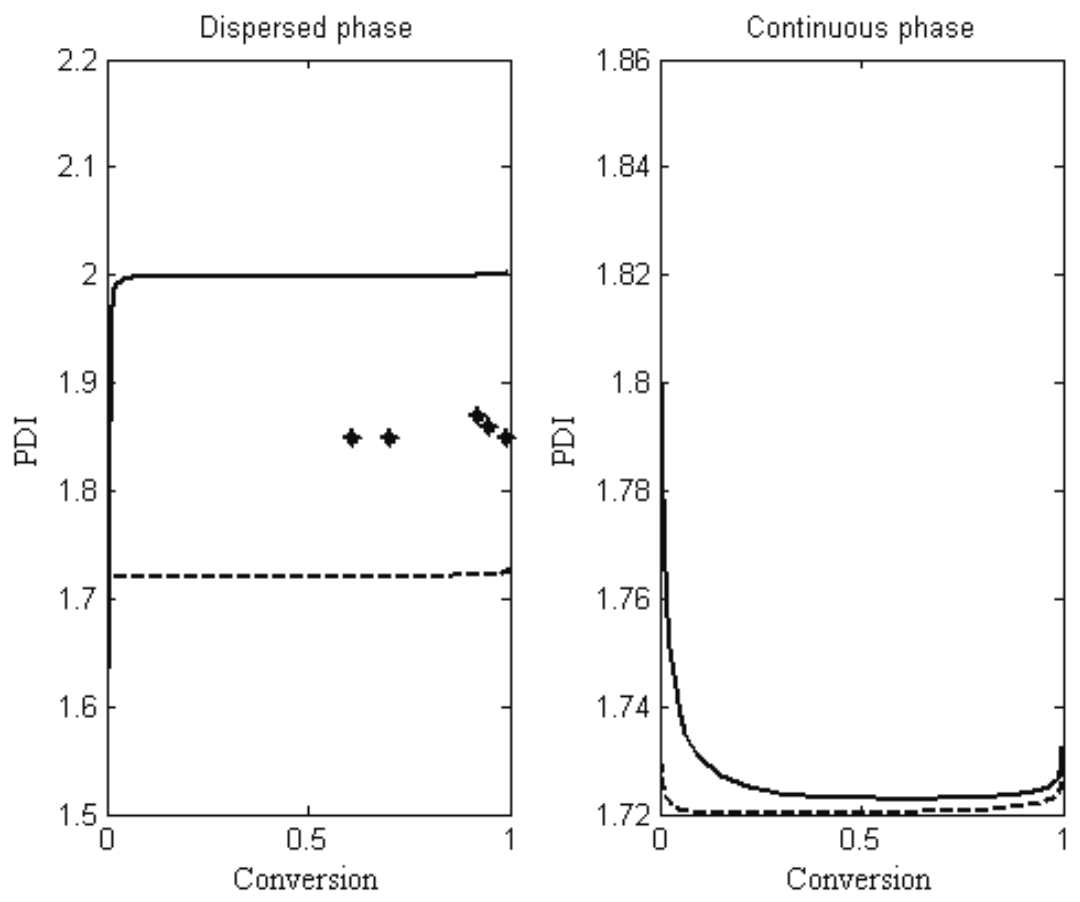

Fig. 4. Poly dispersity index versus monomer conversion. *Experimental results, Case I, --- Case II.

According to Fig. 4, PDI of polymers in the dispersed phase is equal to 2 . This value corresponds to radical polymerization mechanism when $k_{t c}=0$. The PDI values for continuous phase is lower than 2. Because macroradicals do not propagate more than $\mathrm{kcr}$, the concentration of polymer chains with $\mathrm{kcr}$ in the continuous phase is more than that of chain length in the homogeneous polymerization; then PDI of polymers formed in the continuous phase is less than that of PDI in homogeneous radical polymerization. If $\mathrm{k}_{\mathrm{td}}=0$, the $\mathrm{PDI}$ of polymers in the dispersed phase will be 1.5 and for continuous phase will be less than 1.5. The zero PDI content at zero monomer conversion is due to zero initial condition in differential equations (33) and (34). 
According to Fig 4, the PDI of polymer formed in the dispersed phase that is calculated by finite moment method is more than the PDI that was measured qualitatively by experimental data. Moment method is unable to calculate the concentration of precipitated polymer chains in the continuous phase (we will solve this problem in the next paper by a new method) these precipitated polymer chains transfer from the continuous to the dispersed phase to form primary particles [1]. Therefore, the PDI of polymeric product is less than that of PDI that is calculated by moment method.
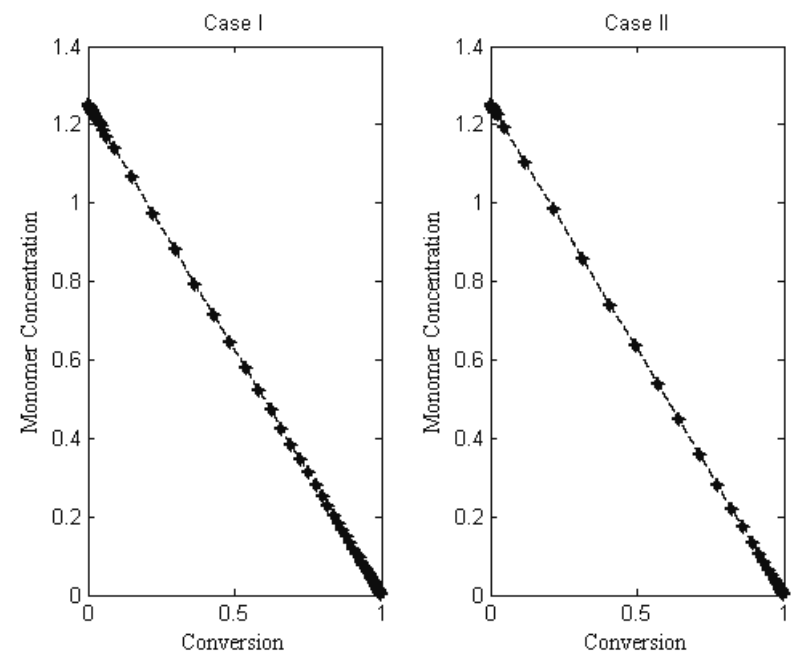

Fig. 5. Initiator concentration versus conversion. * Overall Concentration, --- in the continuous phase, ... in the dispersed phase.

Overall initiator and monomer concentrations of system, initiator and monomer concentration in the continuous and dispersed phases versus monomer conversion are shown in Fig. 5 and 6. According to these figures, the rate of initiator consumption are the same in both phases and equal to the rate of initiator consumption of system as, partition coefficients of initiator are unity. This result is shown for the rate of monomer consumption in both phases too.
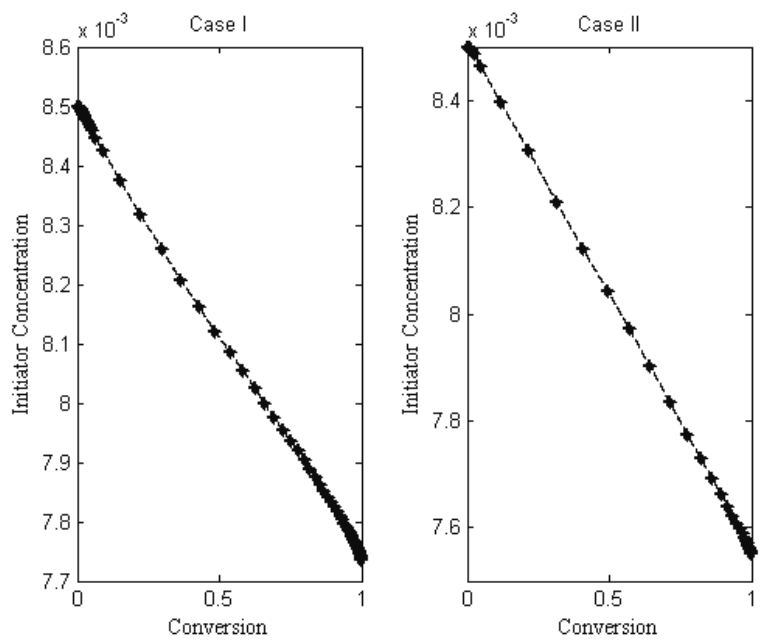

Fig. 6. Monomer concentration versus conversion.* Overall Concentration, --- in the continuous phase, ... in the dispersed phase. 
Fig. 7 shows plots of volume fraction of dispersed phase versus monomer conversion. Primary particles (primary nucleus) are formed at the beginning of polymerization but secondary particles are formed at monomer conversion of 0.5 (polymerization time, $250 \mathrm{sec}$ ). As a result aggregation steps are initiated from monomer conversion value of 0.5 . Formation of primary particles is stopped at conversion value of 0.9 and dropped because of aggregation and formation of secondary particles. As a result, seed stage is stopped at monomer conversion value of 0.5 and the volume fraction of secondary particles is increased rapidly. Therefore at the end of polymerization main polymer particles are of cluster shape.
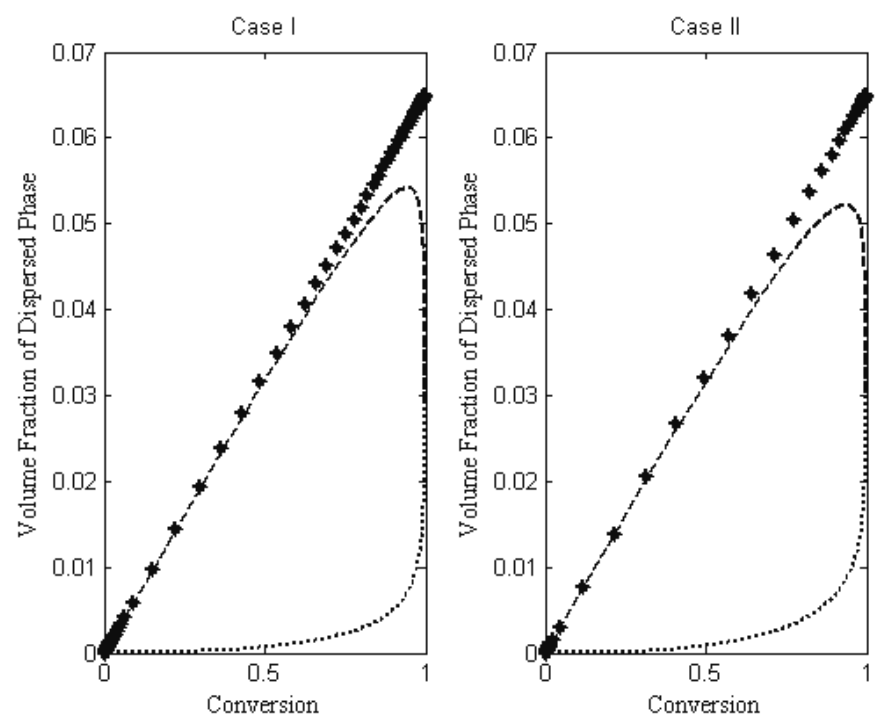

Fig. 7. Volume fraction of dispersed phase versus of monomer conversion. * Volume fraction of dispersed phase, --- volume fraction of primary particles, .....volume fraction of secondary particles.

\section{Conclusions}

This model is able to predict conversion of precipitation polymerization. The deviation from experimental results is due to the purification process. The polymer chains with chain length higher than ' $k c r$ ' in case I are more than that of polymer chains in case II. Then the deviation between modeling and experimental results for case $\mathrm{I}$ is more than that of deviation for case II. In this study, the number and weight average degree of polymerization are calculated separately and then PDI is calculated. This model is able to calculate separately number -and weight average degree of polymerization of polymer formed in both dispersed and continuous phases. Experimental results are fitted with number -and weight average degree of polymerization in the dispersed phase in case I but these is not fitted with that of properties in the continuous phase. Weight average degree of polymerization in the dispersed and continuous phases are similar in case II and do not predict experimental results. Then, case $\|$ is not reasonable prediction for precipitation polymerization of water soluble monomer such as acrylic acid and acrylamide. PDI of polymer formed in the dispersed phase is equal to 2 . This value corresponds to radical polymerization mechanism when $\mathrm{k}_{\mathrm{tc}}=0$. PDI in the continuous phase is less than 2. Primary particles (primary nucleus) are formed at the beginning of polymerization but secondary particles are formed at a monomer conversion of 0.5 . 
As a result, aggregation steps are initiated from conversion value of 0.5 . Formation of primary particles stops at monomer conversion of 0.9 and drop because of aggregation and formation of secondary particles. As a result, seed stage is stopped at this conversion value and the volume fraction of secondary particles increases rapidly.

\section{Experimental}

\section{Reagents}

Acrylic acid, inhibited with hydroquinone monomethyl ether (Fluka) was used as monomer. The monomer was not purified prior to polymerization. Toluene (Merck) was used as polymerization diluent. The initiator, 2,2'-azobis(2,4-dimethylvaleronitrile) (ADVN) was purified before reaction. Sodium hydroxide (Merck) was used as neutralizing agent and distilled water was used as polymer solvent for viscometer experiments.

\section{Apparatus and procedures}

Precipitation polymerization was carried out in a three necked balloon (100 cc) with a condenser. Water bath and a hot plate were used to heat the reactor. Agitation was supplied with a magnetic stirrer. The maximum stirring speed was $100 \mathrm{rpm}$. The temperature inside the reactor was measured by a conventional thermometer. The prescribed amount of monomer was dissolved in the solvent $(50 \mathrm{ml})$ and the solution was then charged into the balloon and nitrogen was bubbled for 20 min before polymerization to remove oxygen. After the oxygen purging was completed, the initiator solution was injected into the reactor to start the polymerization; the reaction were conducted under isothermal condition at about $52 \pm 1^{\circ} \mathrm{C}$. The polymerization reactions were quenched by adding $1 \mathrm{ml}$ of a $1 \%$ hydroquinone solution in methanol at the end of reaction time and were then immersed in an ice- water mixture for 10 $\min$.

\section{Purification process of product}

The products were then filtered, rewashed by its diluent and kept in a vacuum oven at $50{ }^{\circ} \mathrm{C}$ for $12 \mathrm{~h}$.

\section{Conversion measurements}

The conversion from the monomer to the polymer was measured using a gravimetric method.

\section{Molecular weights measurements}

Molecular weight measurements were carried out by Ubbelohde dilution viscometer. The number average and the weight average molecular weights of precipitated polymers were calculated by these equations [27].

$[\eta]_{1}=15.47 \times 10^{-3} \bar{M}_{n}^{0.9}$

The polymer solvent was aq. $\mathrm{NaCl}(1 \mathrm{M})$ and the viscometery was carried out at $25^{\circ} \mathrm{C}$ $[\eta]_{2}=124 \times 10^{-3} \bar{M}_{w}^{0.5}$

The polymer solvent was aq. $\mathrm{NaBr}(0.5 \mathrm{M})$ and the viscometery was carried out at 15 ${ }^{\circ} \mathrm{C}$ 
Then polydispersity index (PDI) was measured qualitatively as;

$P D I=\frac{\bar{M}_{w}}{\bar{M}_{n}}$

\section{Notations}

c Continuous phase

$d \quad$ Dispersed phase

I Initiator

[I] Initiator concentration, $\mathrm{mol} / \mathrm{lit}$

$k c r \quad$ Critical chain length for solubility of macroradicals in the continuous phase

$k_{d} \quad$ Rate constant for the thermal decomposition of initiator, $(\mathrm{min})^{-1}$

$k_{d}^{\prime} \quad$ Rate constant for the monomer- enhanced decomposition of initiator, L/mol.min

$k_{p} \quad$ Rate constant for the chain propagation, L/mol.min

$k_{t c} \quad$ Rate constant for the chain termination by combination, L/mol.min

$k_{t d} \quad$ Rate constant for the chain termination by disproportionation, L/mol.min

$k_{t r, M} \quad$ Rate constant for the chain transfer to the monomer, L/mol.min

$k_{t r, S} \quad$ Rate constant for the chain transfer to the solvent, L/mol.min

$k_{v} \quad$ Rate constant for the change of particle kind, $(\min )^{-1}$

M Monomer

[M] Monomer concentration, mol/lit

$\bar{M}_{n} \quad$ Number average molecular weight, $\mathrm{g} / \mathrm{mol}$

$\bar{M}_{\text {w }} \quad$ Weight average molecular weight, $\mathrm{g} / \mathrm{mol}$

$p \quad$ Monomer conversion

PDI Poly Dispersity Index

$P_{i} \quad$ Dead polymer containing $i$ monomer unit

$R_{0}^{*} \quad$ Primary radical

$R_{i}^{*} \quad$ Macroradical containing $i$ monomer unit

$\Re_{i} \quad$ Rate of initiation, mol/lit min

$S \quad$ Solvent

$V_{P}^{\prime} \quad$ Molar volume of polymer, $\mathrm{L} / \mathrm{mol}$

$\bar{X}_{n} \quad$ Number average degree of polymerization

$\bar{X}_{w} \quad$ Weight average degree of polymerization 
$\phi_{1} \quad$ Volume fraction of primary particles

$\phi_{2} \quad$ Volume fraction of secondary particles

$\phi_{c} \quad$ Volume fraction of continuous phase

$\phi_{d} \quad$ Volume fraction of dispersed phase

$\varphi \quad$ Partition coefficient between continuous and dispersed phase

$[\eta] \quad$ Intrinsic viscosity, L/g

$\lambda \quad$ Conventional moment for macroradicals

$\lambda^{*} \quad$ Finite moment for macroradicals

$\mu \quad$ Conventional moment for dead polymers

$\mu^{*} \quad$ Finite moment for dead polymers

$\varsigma \quad$ Probability of propagation reaction

\section{References}

[1] Guyot, A. "Precipitation Polymerization", In: Comprehensive Polymer Science, Allen S. G., $1^{\text {st }}$ edition, Pergamon Press, London, 1989, Vol. 4 P. 261.

[2] Guyot, A. "Precipitation Polymerization", In: Polymeric Material Encyclopedia, Salamone J. C., $1^{\text {st }}$ edition., CRC Press, Boca Raton, 1996, Vol. 9 P. 7228.

[3] Mi, Y.; Wang, J.; Zhang, Y.; Chen, E.; Chang, S. Z. D. Polymer, 2001, 42, 4533.

[4] Jewrajka, S. K.; Mandal, B. M. Macromolecules, 2003, 36, 311-317.

[5] Hunkeler, D.; Hamielec, A. E.; Baade, W. Polymer, 1989, 30, 127.

[6] Inchausti, I.; Sasia, P. M.; Kataime, I. Journal of Material Science, 2005, 40, 4833.

[7] Saraf, M. K.; Gerard, S.; Wojcinski, L. M.; Charpentier, P. A.; Desimone, J. M.; Robert, G. W. Macromolecules, 2002, 35, 7976.

[8] Ahmed, T.S.; DeSimone, J. M.; Robert, G. W. Chemical Engineering Science, 2004, 59, 5139.

[9] Chernyshev, A. V.; Soini, A. E.; Surovtsev, I. V.; Maltsev, V. P.; Soini, E. J. Polymer Sci., Polymer Chem., 1997, 35, 1799.

[10] Chernyshev, A. V.; Soini, A. E.; Maltsev, V. P.; Soini, E. Macromolecules, 1998, 31,6455 .

[11] Nishida, R.; Poehlein, G. W.; Schork, F. J. Polymer Reaction Engineering, 1995, 3, 397.

[12] Liu, T.; DeSimone, J. M.; Roberts, G. W. Chemical Engineering Science, 2006, $61,3129$.

[13] Avela, A.; Poersch, H.; Reichert, K. Die Angewandte Makromol. Chem., 1990, $175,107$.

[14] Poersch, H.; Avela, A.; Reichert, K. Die Angewandte Makromol. Chem., 1993, 206, 157.

[15] Barret, K. E. J.; Thomas, H. R. J. Polymer Sci., Part A, 1969, 7, 2621.

[16] Charpentier, P. A.; DeSimone, J. M.; Robert, G. W. Industrial \& Engineering Chem. Research, 2000, 39, 4588.

[17] Muller, P. A.; Storti, G.; Morbidelli, M.; Apostolo, M.; Mrtin, R. Macromolecules, 2005, 38, 7150-7163. 
[18] Yasuda, M.; Seki, H.; Yokoyama, H.; Ogino, H.; Ishimi, K.; Ishigawa, H. Macromolecules, 2001, 34, 3261.

[19] Chatzidoukas, C.; Pladis, P.; Kiparissides, C. Ind. Eng. Chem. Res., 2003, 42, 743.

[20] Bunyakan, C.; Armanet, L.; Hunkeler, D. Polymer, 1999, 40, 6225.

[21] Bamford, G.H. "Radical Polymerization", In: Encyclopedia of Polymer Science and Engineering , $2^{\text {st. }}$ edition, Kroschwitz J. I., John Wiley \& Sons, New York, 1988, Vol. 13 P. 708.

[22] Bunyakan, C.; Hunkeler, D. Polymer, 1999, 40, 6213.

[23] Gromov, V. F.; Galperina, N. I.; Osmanov, T. O.; Khomikovskii, P. M.; Abkin, A. D. Europ. Polym. J., 1980, 16, 529.

[24] Chapiro, A.; Dulieu, J. Europ. Polym. J., 1977, 13, 563.

[25] Crowley, T.J.; Choi, K.Y. Industrial \& Engineering Chem. Research, 1997, 36, 1419.

[26] Crowley, T. J.; Choi, K.Y. J. Appl. Polymer Sci., 1998, 70, 1017.

[27] Kurata, M.; Tsunashima, Y. Viscosity- Molecular Weight Relationships and Unperturbed Dimensions of Linear Chain Molecules. In: Polymer Handbook, Brandrupt E. H., Immergut E. A., Grulke J., $4^{\text {th }}$ edn., John Wiley \& Sons, New York, 1999, P: VII1. 